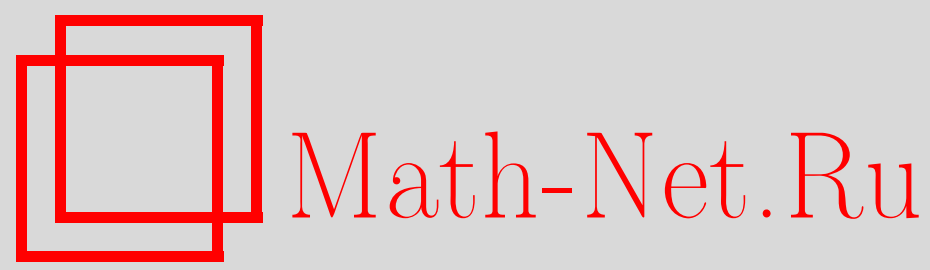

И. Я. Новиков, Асимптотика корней полиномов Бернштейна, используемых в построении модифицированных всплесков Добеши, Матем. заметки, 2002, том 71, выпуск 2, 239 253

DOI: https://doi.org/10.4213/mzm343

Использование Общероссийского математического портала Math-Net.Ru подразумевает, что вы прочитали и согласны с пользовательским соглашением http://www.mathnet.ru/rus/agreement

Параметры загрузки:

IP : 54.196 .121 .252

26 апреля 2023 г., 13:36:34

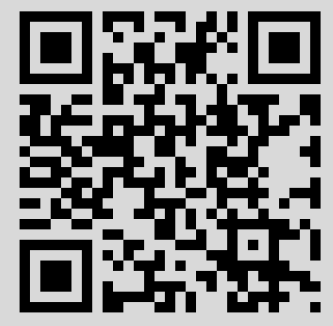




\section{АСИМПТОТИКА КОРНЕЙ ПОЛИНОМОВ БЕРНШТЕЙНА, ИСПОЛЬЗУЕМЫХ В ПОСТРОЕНИИ МОДИФИЦИРОВАННЫХ ВСПЛЕСКОВ ДОБЕШИ}

\section{И. Я. Новиков}

Статья посвящена изучению асимптотики корней последовательности полиномов Бернштейна, приближающих кусочно-линейную функцию. Эта последовательность возникает при построении модифицированных всплесков с компактным носителем, которые, в отличие от классических всплесков Добеши, сохраняют локализацию с ростом гладкости. Доказано, что предельной кривой для корней является граница области сходимости рассматриваемых полиномов Бернштейна на комплексной плоскости.

Библиографияя: 13 названий.

1. Введение. Всплеском назьвают функцию $\psi$, сжатия и сдвиги которой $\left\{\psi_{j k}(t):=\right.$ $\left.2^{j / 2} \psi\left(2^{j} t-k\right)\right\}_{j, k \in \mathbb{Z}}$ образуют ортонормированньй базис в $L^{2}(\mathbb{R})$. Всплески с компактным носителем и произвольной конечной гладкостью построены Добеши в [1]. Существенньм недостатком этой конструкции является ухудшение локализованности всплесков с возрастанием гладкости (см. [2]). В статьях автора [3], [4] построены модифицированные всплески Добеши, сохраняющие локализацию с возрастанием гладкости. В построении используются полиномы Бернштейна на $B$ [5], аппроксимирующие кусочно-аналитическую функцию на отрезке $[-1,1]$. Эти полиномы определяют квадрат модуля тригонометрического полинома $m$ (масштабирующего фильтра), являющегося центральным элементом конструкции всплесков с компактным носителем. Полином $m$ находится при помощи леммы Рисса [6]. Для ее применения необходимо знать корни полинома $B$. Более того, полином $m$ определяется таким способом неоднозначно. Информация о корнях полинома $B$ позволяет выбирать $m$ с дополнительными свойствами, определяюшими важные для приложений характеристики соответствуюших всплесков: степень асимметричности, локализованность, гладкость (см., например, [7]).

Данная статья посвящена исследованию асимптотики корней следующей последовательности многочленов Бернштейна:

$$
B_{L}(x)=\sum_{l=0}^{L-1}\left(\begin{array}{c}
4 L \\
l
\end{array}\right) x^{l}(1-x)^{4 L-l}+\sum_{l=L}^{3 L-1}\left(\frac{3}{2}-\frac{l}{2 L}\right)\left(\begin{array}{c}
4 L \\
l
\end{array}\right) x^{l}(1-x)^{4 L-l}, \quad L \in \mathbb{N},
$$

Работа выполнена при частичной финансовой поддержке Российского фонда фундаментальных исследований, грант № 98-01-00044, и программы "Университеты России". 
аппроксимирующих на $[0,1]$ кусочно-линейную функцию

$$
f(t):= \begin{cases}1, & t \in\left[0, \frac{1}{4}\right], \\ \frac{3}{2}-2 t, & t \in\left(\frac{1}{4}, \frac{3}{4}\right], \\ 0, & t \in\left(\frac{3}{4}, 1\right] .\end{cases}
$$

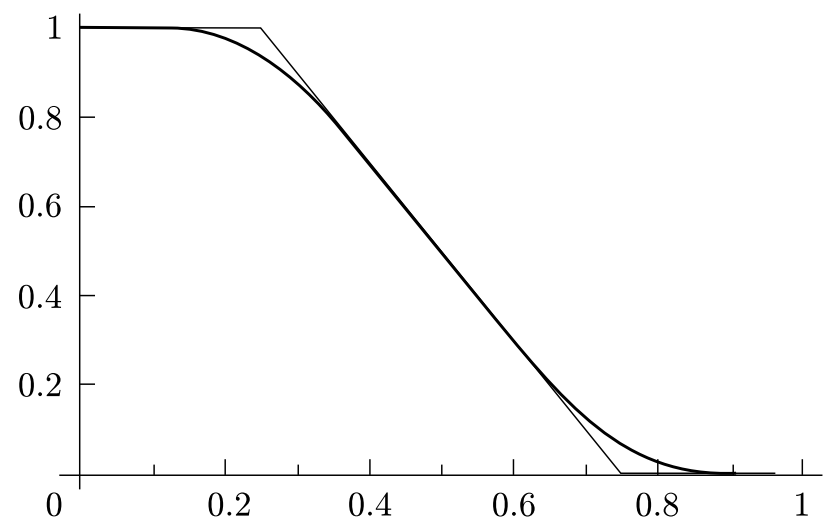

Рис. 1

На рис. 1 показаны функция $f$ и полином Бернштейна $B_{7}$. Подобная задача для многочленов Бернштейна, аппроксимирующих на $[0,1]$ характеристическую функцию отрезка $[0,1 / 2]$ и соответствуюших конструкции классических всплесков Добеши, изучалась в [8]-[10]. Используя методы [4], можно доказать, что модифицированные всплески Добеши, построенные таким же образом, как в [3], с заменой использованной там кусочно-аналитической функции на кусочно-линейную, сохраняют локализацию с ростом гладкости. Рисунок 2 показывает корни $B_{37}$. Цель работы - найти кривую, на которой расположены корни, и исследовать поведение корней при $L \rightarrow \infty$. Оказывается, что предельная кривая состоит из частей двух лемнискат. Первая лемниската определяется уравнением

$$
\mathscr{L}_{1}:=\left\{x:\left|\frac{x}{1 / 4}\right|^{1 / 4}\left|\frac{1-x}{3 / 4}\right|^{3 / 4}=1\right\},
$$

вторая -

$$
\mathscr{L}_{2}:=\left\{x:\left|\frac{x}{3 / 4}\right|^{3 / 4}\left|\frac{1-x}{1 / 4}\right|^{1 / 4}=1\right\}
$$

(рис. 3).

Рисунок 4 показьвает нули $B_{37}$ вместе с $\mathscr{L}_{1}$ и $\mathscr{L}_{2}$. Маленькая окружность на рис. 4 имеет центр в $36 / 35$ и радиус $6 / 35$. Доказано, что многочлены $B_{L}$ не имеют корней, не равных 1 , внутри этого круга. В п. 2 доказьваются точные результаты относительно корней $B_{L}$, содержащихся в левой петле лемнискаты $\mathscr{L}_{2}$ и имеющих вещественную часть меньшую чем $1 / 4$. Подобные результаты получены относительно других областей внутри петель лемнискат $\mathscr{L}_{1}$ и $\mathscr{L}_{2}$ (они не приводятся здесь из-за ограничения объема). Вспомогательные утверждения вынесены в отдельный пункт - п. 3. 


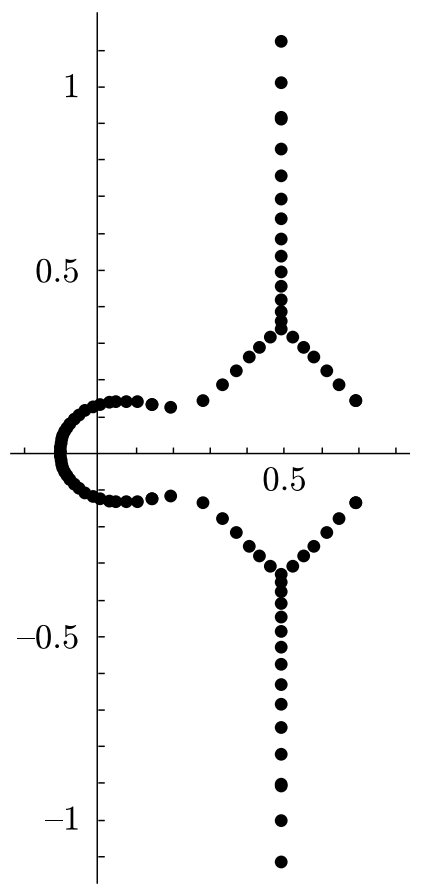

Рис. 2. Нули $B_{37}$
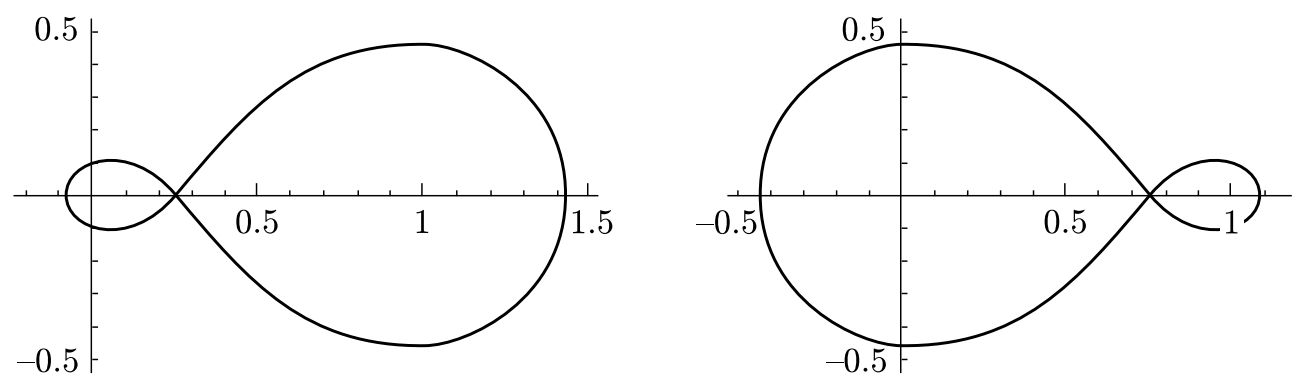

Рис. 3. Лемнискаты $\mathscr{L}_{1}$ и $\mathscr{L}_{2}$

Численные эксперименты показывают, что вертикальная прямая линия $\operatorname{Re} x=1 / 2$ является предельной кривой для нулей $B_{L}$, расположеных вне лемнискат. Этот факт нуждается в теоретическом обосновании.

Полученные результаты указывают, что расположение корней полиномов Бернштейна, аппроксимируюших кусочно-аналитическую функцию, подобно расположению корней частных сумм степенного ряда. Известно (см. [11]), что любая граничная точка круга сходимости степенного ряда является предельной для множества корней частных сумм этого ряда. Из результатов статьи [12] следует, что последовательность $\left\{B_{L}\right\}_{L=1}^{\infty}$ сходится в комплексной области на пересечении внутренностей лемнискат $\mathscr{L}_{1}$ и $\mathscr{L}_{2}$. Таким образом, в рассмотренном случае предельной кривой для корней полиномов Бернштейна так же, как и для частных сумм степенного ряда, является граница области сходимости. 


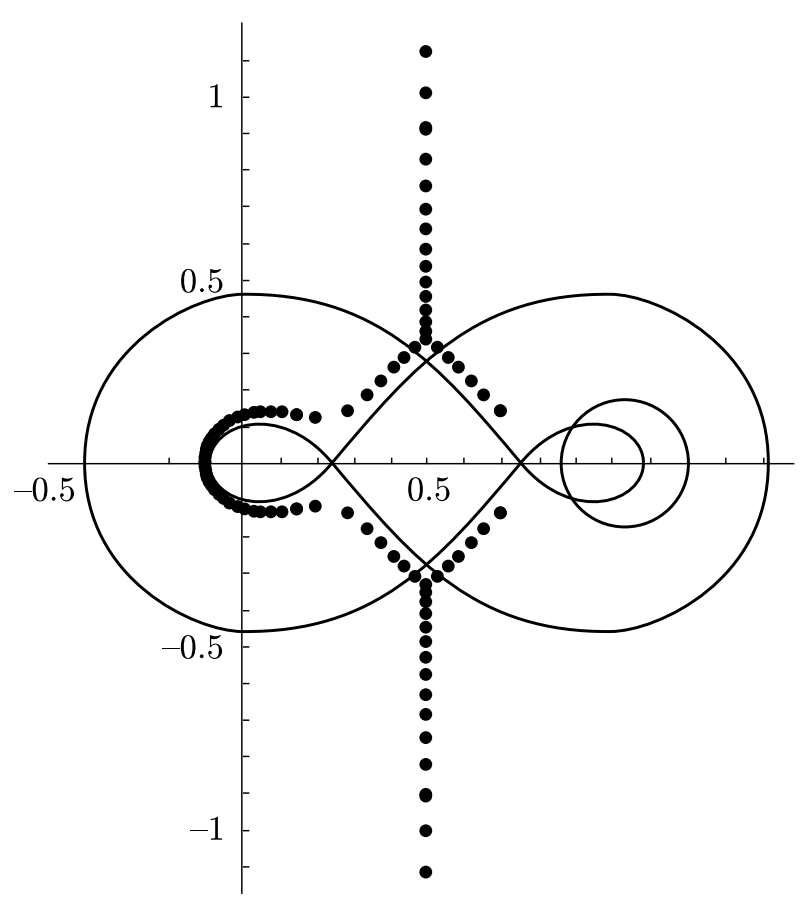

Рис. 4. Корни $B_{37}$ и лемнискаты

\section{2. Основные результаты.}

Tеорема 2.1. Все корни многочленов $B_{L}, L \in \mathbb{N}$, не равные 1 , лежат вне окружности

$$
\left|x-\frac{36}{35}\right|=\frac{6}{35} .
$$

ДокаЗАТЕЛЬСтво. Ясно, что для $x \neq 1$ имеет место равенство

$$
B_{L}(x)=(1-x)^{4 L}\left[\sum_{l=0}^{L-1}\left(\begin{array}{c}
4 L \\
l
\end{array}\right)\left(\frac{x}{1-x}\right)^{l}+\sum_{l=L}^{3 L-1}\left(\frac{3}{2}-\frac{l}{2 L}\right)\left(\begin{array}{c}
4 L \\
l
\end{array}\right)\left(\frac{x}{1-x}\right)^{l}\right]
$$

Пусть $z:=x /(1-x)$. Применим теорему Энестрема-Какея [6, с. 114, проблема 23] к полиному

$$
\sum_{l=0}^{L-1}\left(\begin{array}{c}
4 L \\
l
\end{array}\right) z^{l}+\sum_{l=L}^{3 L-1}\left(\frac{3}{2}-\frac{l}{2 L}\right)\left(\begin{array}{c}
4 L \\
l
\end{array}\right) z^{l}
$$


На основании этой теоремы корни находятся в кольце

$$
\begin{aligned}
& \min \left\{\min _{0 \leqslant l \leqslant L-1} \frac{\left(\begin{array}{c}
4 L \\
l
\end{array}\right)}{\left(\begin{array}{c}
4 L \\
l+1
\end{array}\right)}, \min _{L \leqslant l \leqslant 3 L-2} \frac{\left(\frac{3}{2}-\frac{l}{2 L}\right)\left(\begin{array}{c}
4 L \\
l
\end{array}\right)}{\left(\frac{3}{2}-\frac{l+1}{2 L}\right)\left(\begin{array}{c}
4 L \\
l+1
\end{array}\right)}\right\} \\
& \leqslant|z| \leqslant \max \left\{\max _{0 \leqslant l \leqslant L-1} \frac{\left(\begin{array}{c}
4 L \\
l
\end{array}\right)}{\left(\begin{array}{c}
4 L \\
l+1
\end{array}\right)}, \max _{L \leqslant l \leqslant 3 L-2} \frac{\left(\frac{3}{2}-\frac{l}{2 L}\right)\left(\begin{array}{c}
4 L \\
l
\end{array}\right)}{\left(\frac{3}{2}-\frac{l+1}{2 L}\right)\left(\begin{array}{c}
4 L \\
l+1
\end{array}\right)}\right\} .
\end{aligned}
$$

Прямые вычисления показывают, что $1 /(4 L) \leqslant|z| \leqslant 6-14 /(L+2)<6$. В терминах переменной $x$ имеем

$$
\left\{x:\left|\frac{x}{1-x}\right|<6\right\}=\left\{x:\left|x-\frac{36}{35}\right|>\frac{6}{35}\right\} \text {. }
$$

Пусть $\alpha_{1}$ и $\beta_{1}$ - области внутри левой и правой петли лемнискаты $\mathscr{L}_{1} ; \alpha_{2}$ и $\beta_{2}$ - то же самое для $\mathscr{L}_{2}$ :

$$
\begin{aligned}
& \alpha_{1}:=\left\{x:\left|\frac{x}{1 / 4}\right|^{1 / 4}\left|\frac{1-x}{3 / 4}\right|^{3 / 4}<1, \operatorname{Re} x<\frac{1}{4}\right\}, \\
& \beta_{1}:=\left\{x:\left|\frac{x}{1 / 4}\right|^{1 / 4}\left|\frac{1-x}{3 / 4}\right|^{3 / 4}<1, \operatorname{Re} x>\frac{1}{4}\right\}, \\
& \alpha_{2}:=\left\{x:\left|\frac{x}{3 / 4}\right|^{3 / 4}\left|\frac{1-x}{1 / 4}\right|^{1 / 4}<1, \operatorname{Re} x<\frac{3}{4}\right\}, \\
& \beta_{2}:=\left\{x:\left|\frac{x}{3 / 4}\right|^{3 / 4}\left|\frac{1-x}{1 / 4}\right|^{1 / 4}<1, \operatorname{Re} x>\frac{3}{4}\right\} .
\end{aligned}
$$

Tеорема 2.2. Корни многочленов $B_{L}(x), L \in \mathbb{N}$, находящиеся в $\{x:|x| \leqslant 1 / 4\}$, удовлетворяют неравенству

$$
\left|\frac{x}{1 / 4}\right|^{1 / 4}\left|\frac{1-x}{3 / 4}\right|^{3 / 4}>a(L)
$$

əде

$$
\begin{gathered}
a(L)=\left(1-\left(\frac{5}{27}\right)^{L} \frac{9}{128 L}\right)^{3 /(12 L+4)}\left(\frac{\sqrt{3}}{4 \sqrt{2 \pi L}} \Phi(L)\right)^{-3 /(12 L+4)} \\
\Phi(L):=\frac{\Gamma^{*}(4 L)}{\Gamma^{*}(L) \Gamma^{*}(3 L)}, \quad \Gamma^{*}(r):=\sqrt{\frac{r}{2 \pi}} e^{r} r^{-r} \Gamma(r) .
\end{gathered}
$$


ДоКАЗАТЕЛЬСТво. Пусть

$$
\begin{gathered}
b_{N, l}(x):=\left(\begin{array}{c}
N \\
l
\end{array}\right) x^{l}(1-x)^{N-l}, \quad l=0,1, \ldots, N, \quad B_{L, 1}(x):=\sum_{l=0}^{3 L-1}\left(\frac{3}{2}-\frac{l}{2 L}\right) b_{4 L, l}(x), \\
R_{L, 1}(x):=\sum_{l=3 L+1}^{4 L}\left(\frac{l}{2 L}-\frac{3}{2}\right) b_{4 L, l}(x), \quad B_{L, 2}(x):=\sum_{l=0}^{L-1}\left(\frac{1}{2}-\frac{l}{2 L}\right) b_{4 L, l}(x) .
\end{gathered}
$$

Известно, что

$$
\sum_{l=0}^{N} b_{N, l}(x)=1, \quad \sum_{l=0}^{N} \frac{l}{N} b_{N, l}(x)=x .
$$

Поэтому

$$
B_{L}(x)=B_{L, 1}(x)-B_{L, 2}(x)=\frac{3}{2}-2 x+R_{L, 1}(x)-B_{L, 2}(x) .
$$

Применяя к $B_{L, 2}$ формулы (3.3) и (3.6), получаем, что

$$
B_{L, 2}(x)=\frac{1}{2}-2 x+6 L\left(\begin{array}{c}
4 L-1 \\
L-1
\end{array}\right) I_{4 L+1, L-1}(x) x^{L+1}(1-x)^{3 L+1},
$$

где

$$
I_{N, k}(x):=\int_{0}^{1} s(1-x s)^{-N}(1-s)^{k} d s .
$$

Теперь (2.4) и (2.5) влекут

$$
B_{L}(x)=1+R_{L, 1}(x)-6 L\left(\begin{array}{c}
4 L-1 \\
L-1
\end{array}\right) I_{4 L+1, L-1}(x) x^{L+1}(1-x)^{3 L+1} .
$$

Очевидно, что для $|x| \leqslant 1 / 4$ и $s \in[0,1]$

$$
\left|(1-x s)^{-1}\right| \leqslant\left(1-\frac{s}{4}\right)^{-1}
$$

На основании (2.6) и (2.7), если $B_{L}(x)=0$ и $|x| \leqslant 1 / 4$, то

$$
\left|\left(1+R_{L, 1}(x)\right) x^{-L-1}(1-x)^{-3 L-1}\right|<6 L\left(\begin{array}{c}
4 L-1 \\
L-1
\end{array}\right) I_{4 L+1, L-1}\left(\frac{1}{4}\right) .
$$

Из (2.5) следует, что

$$
6 L\left(\begin{array}{c}
4 L-1 \\
L-1
\end{array}\right) I_{4 L+1, L-1}\left(\frac{1}{4}\right)=B_{L, 2}\left(\frac{1}{4}\right)\left(\frac{1}{4}\right)^{-L-1}\left(\frac{3}{4}\right)^{-3 L-1} .
$$

Теперь (2.8) и (2.9) влекут

$$
\left|\frac{x}{1 / 4}\right|^{L+1}\left|\frac{1-x}{3 / 4}\right|^{3 L+1}>\frac{\left|1+R_{L, 1}(x)\right|}{B_{L, 2}(1 / 4)} .
$$


Учитьвая следствие 3.3 и (3.11), получаем

$$
\left|\frac{x}{1 / 4}\right|^{L+1}\left|\frac{1-x}{3 / 4}\right|^{3 L+1}>\frac{1-\left(\frac{5}{27}\right)^{L} \frac{9}{128 L}}{\frac{\sqrt{3}}{4 \sqrt{2 \pi L}} \Phi(L)} .
$$

Возводя обе части последнего неравенства в степень $3 /(4(3 L+1))$, получаем $(2.2)$.

Пусть $\mathscr{L}_{11}$ обозначает лемнискату

$$
\mathscr{L}_{11}(L):=\left\{x:\left|\frac{x}{1 / 4}\right|^{1 / 4}\left|\frac{1-x}{3 / 4}\right|^{3 / 4}=a(L)\right\} .
$$

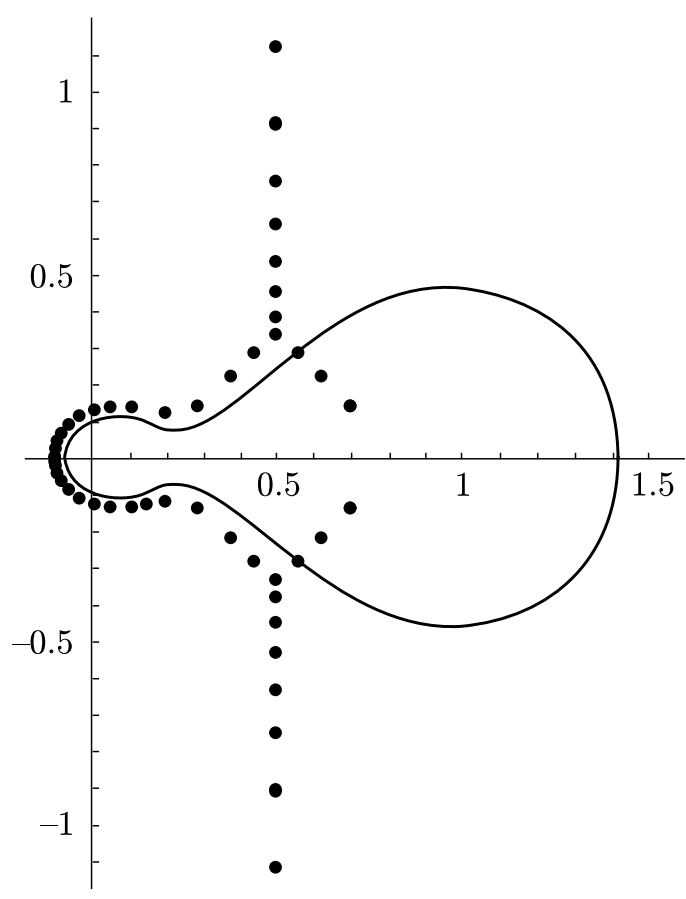

Рис. 5

На рис. 5 изображены корни $B_{20}$ и лемниската $\mathscr{L}_{11}(20)$.

Зафиксируем теперь произвольное $\delta>0$ и рассмотрим область

$$
\alpha_{2,1}:=\alpha_{2} \cap\left\{\left|x-\frac{1}{4}\right|>\delta\right\} \cap\left\{\operatorname{Re} x<\frac{1}{4}\right\} .
$$

В силу следствия 3.5

$$
I_{4 L+1, L-1}(x)=\frac{1}{L^{2}(1-4 x)^{2}}+O\left(L^{-3}\right) .
$$


Если $B_{L}(x)=0$, то в силу $(2.6)$

$$
x^{-L-1}(1-x)^{-3 L-1}=\frac{6 L\left(\begin{array}{c}
4 L-1 \\
L-1
\end{array}\right) I_{4 L+1, L-1}(x)}{1+R_{L, 1}(x)} .
$$

По формуле Стирлинга

$$
\left(\begin{array}{c}
4 L-1 \\
L-1
\end{array}\right) \sim \frac{4^{4 L}}{2 \sqrt{6 \pi L} 3^{3 L}} .
$$

Поэтому (2.11), (2.10) и следствие 3.3 влекут

$$
x^{-L-1}(1-x)^{-3 L-1}=\frac{4^{4 L}}{\sqrt{6 \pi L} L 3^{3 L-1}} \frac{1}{(1-4 x)^{2}}\left(1+O\left(L^{-1}\right)\right) .
$$

Возводя обе части этого неравенства в степень $(-1 /(4 L+4))$, получаем, что корень $x$ многочлена $B_{L}$ удовлетворяет условию

$$
\left|\frac{x}{1 / 4}\right|^{1 / 4}\left|\frac{1-x}{3 / 4}\right|^{3 / 4}=\left(\frac{2^{17} \pi L^{3}}{3^{7}}\right)^{1 /(8(L+1))}(|1-x| \cdot|1-4 x|)^{1 /(2(L+1))}\left(1+O\left(L^{-2}\right)\right) .
$$

Из этого следует

Теорема 2.3. Пусть $\delta>0, L \in \mathbb{N}$. Тогда расстояние от любого корня полинома $B_{L}(x)$, содержашегося в $\alpha_{2,1}$, до кривой $\mathscr{L}_{1, L}$ :

$$
\left|\frac{x}{1 / 4}\right|^{1 / 4}\left|\frac{1-x}{3 / 4}\right|^{3 / 4}=\left(\frac{2^{17} \pi L^{3}}{3^{7}}\right)^{1 /(8(L+1))}(|1-x| \cdot|1-4 x|)^{1 /(2(L+1))},
$$

не превосходит $c(\delta) L^{-2}$.

Рисунок 6 показьвает корни $B_{20}$ и кривую $\mathscr{L}_{1, L}$ внутри области $\alpha_{2,1}$.

ДокАЗАТЕЛЬСтво. Пусть $X-$ корень $B_{L}$, расположенньй в $\alpha_{2,1}$, а $x$ - точкана $\mathscr{L}_{1, L}$, ближайшая к $X, \varepsilon:=x-X$. Так как $|1+\varepsilon|^{a}=1+O(a|\varepsilon|)$ для любого $a \in(0,1)$, то

$$
\begin{gathered}
|1-X|^{2 /(L+1)}=|1-x|^{2 /(L+1)} \cdot\left|1+\frac{\varepsilon}{1-x}\right|^{2 /(L+1)}=|1-x|^{2 /(L+1)}\left(1+O\left(\frac{|\varepsilon|}{L}\right)\right), \\
|1-4 X|^{2 /(L+1)}=|1-4 x|^{2 /(L+1)}\left(1+O\left(\frac{|\varepsilon|}{L}\right)\right), \\
\left|X(1-X)^{3}\right|=\left|x(1-x)^{3}\right| \cdot\left|1+\frac{2+x}{x(1-x)} \varepsilon+O\left(\varepsilon^{2}\right)\right| .
\end{gathered}
$$

Пусть $E:=(2+x) /(x(1-x))$. Так как $x \in \mathscr{L}_{1, L}$, то

$$
\begin{aligned}
\frac{\left|\frac{X}{1 / 4}\right| \cdot\left|\frac{1-X}{3 / 4}\right|^{3}}{\left(\frac{2^{17} \pi L^{3}}{3^{7}}\right)^{1 /(2(L+1))}(|1-X| \cdot|1-4 X|)^{1 /(L+1)}} & =\frac{\left|1+E \varepsilon+O\left(\varepsilon^{2}\right)\right|}{1+O(|\varepsilon| / L)} \\
& =|1+E \varepsilon+O(|\varepsilon|)|=1+O\left(L^{-2}\right) .
\end{aligned}
$$




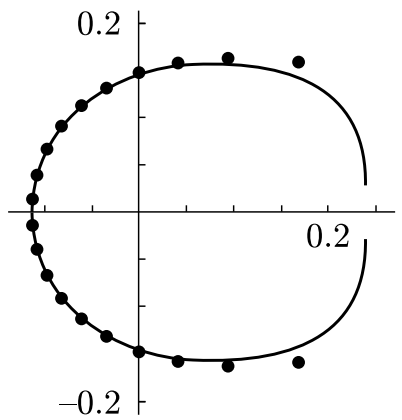

Рис. 6

Последнее равенство следует из (2.12) и влечет, что $\varepsilon=O\left(L^{-2}\right)$.

СлЕДСтвиЕ 2.1. Пусть $\delta>0$. Корни $B_{L}(x), L \in \mathbb{N}$, содержашиеся в $\alpha_{2,1}$, находятся на расстоянии не больше, чем $c^{\prime}(\delta) L^{-1}$, от кривой $\mathscr{L}_{1, L}^{\prime}$, задаваемой уравнением

$$
\left|\frac{x}{1 / 4}\right|^{1 / 4}\left|\frac{1-x}{3 / 4}\right|^{3 / 4}=1+\frac{\log \left(2^{17} \pi L^{3} / 3^{7}\right)}{8 L+1} .
$$

Рисунок 7 показьвает корни $B_{20}$ и $\mathscr{L}_{1,20}^{\prime}$ в $\alpha_{2,1}$.

ДокАЗАТЕЛЬСтво. Утверждение следует из того, что $t=1+\log t+O\left((t-1)^{-2}\right)$.

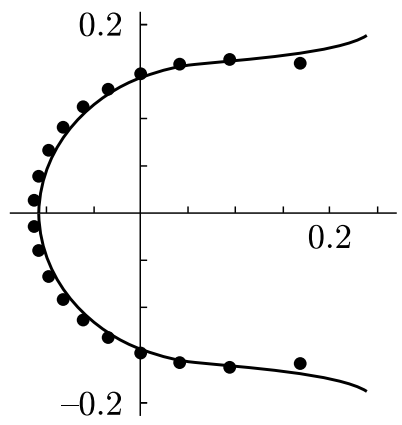

Рис. 7

3. Вспомогательные результаты. Прежде всего представим некоторые специальные многочлены Бернштейна в виде полиномов Тейлора.

Пусть $T_{g, k}(x)$ - полином Тейлора для функции $g$ в точке 0 порядка $k$ :

$$
T_{g, k}(x):=\sum_{l=0}^{k} \frac{g^{(l)}(0)}{l !} x^{l}
$$

Пусть

$$
A_{N, k}(x):=\sum_{l=0}^{k} b_{N, l}(x)
$$


Теорема 3.1. Для любого $N \in \mathbb{N} u$ любого $k \in\{0,1,2, \ldots, N\}$

$$
A_{N, k}(x)=(1-x)^{N-k} T_{h_{N-k}, k}(x),
$$

əде $h_{l}(x):=(1-x)^{-l}$.

ДоКАЗАТЕЛЬСТВО. Ясно, что

$$
h_{l}^{(m)}(0)=l(l+1) \cdots(l+m-1), \quad m \in \mathbb{N} .
$$

Формула (3.1) доказьвается по индукции. Для $k=0$ она очевидна. Предположим, что формула (3.1) верна для $k$. Для $k+1$ имеем

$$
\begin{aligned}
A_{N, k+1}(x) & =A_{N, k}(x)+\left(\begin{array}{c}
N \\
k+1
\end{array}\right) x^{k+1}(1-x)^{N-k-1} \\
& =(1-x)^{N-k} T_{h_{N-k}, k}(x)+\left(\begin{array}{c}
N \\
k+1
\end{array}\right) x^{k+1}(1-x)^{N-k-1} \\
& =(1-x)^{N-k-1}\left(T_{h_{N-k}, k}(x)-x T_{h_{N-k}, k}(x)+\left(\begin{array}{c}
N \\
k+1
\end{array}\right) x^{k+1}\right) .
\end{aligned}
$$

Принимая во внимание (3.2), получаем (3.1) для $k+1$.

СлЕДСТВИЕ 3.1. Для любого $N \in \mathbb{N} u$ любого $k \in\{0,1,2, \ldots, N\}$

$$
A_{N, k}(x)=1-(N-k)\left(\begin{array}{c}
N \\
k
\end{array}\right) \int_{0}^{1}(1-x s)^{-N-1}(1-s)^{k} d s x^{k+1}(1-x)^{N-k}
$$

ДокАЗАТЕЛьСТво. Интегральная формула Тейлора для остаточного члена дает

$$
T_{h_{N-k}, k}(x)=(1-x)^{k-N}-\frac{(N-k) \cdots N}{k !} \int_{0}^{x}(1-s)^{-N-1}(x-s)^{k} d s .
$$

Замена переменных $s=x s$ приводит к (3.3).

Теорема 3.1 и следствие 3.1 являются обобщениями теоремы 1 и равенства (14) из [9]. Пусть

$$
C_{N, k}(x):=\sum_{l=1}^{k} \frac{l}{N} b_{N, l}(x) .
$$

СлЕДСТВИЕ 3.2. Для любого $N \in \mathbb{N} u$ ^юбого $k \in\{1,2, \ldots, N\}$

$$
\begin{gathered}
C_{N, k}(x)=x(1-x)^{N-k} T_{h_{N-k}, k-1}(x) \\
C_{N, k}(x)=x-(N-k)\left(\begin{array}{c}
N-1 \\
k-1
\end{array}\right) \int_{0}^{1}(1-x s)^{-N}(1-s)^{k-1} d s x^{k+1}(1-x)^{N-k} .
\end{gathered}
$$


ДокаЗАТЕЛЬСтво. Так как

$$
\frac{l}{N}\left(\begin{array}{c}
N \\
l
\end{array}\right)=\left(\begin{array}{c}
N-1 \\
l-1
\end{array}\right)
$$

то, используя (3.1), получаем (3.5):

$$
\begin{aligned}
C_{N, k}(x) & =x \sum_{l=1}^{k}\left(\begin{array}{c}
N-1 \\
l-1
\end{array}\right) x^{l-1}(1-x)^{(N-1)-(l-1)} \\
& =x A_{N-1, k-1}(x)=x(1-x)^{N-k} T_{h_{N-k}, k-1}(x) .
\end{aligned}
$$

Так же, как при доказательстве (3.4), имеем

$$
\begin{aligned}
T_{h_{N-k}, k-1}(x) & =(1-x)^{k-N}-\frac{(N-k) \cdots(N-1)}{(k-1) !} \int_{0}^{x}(1-s)^{-N}(x-s)^{k-1} d s \\
& =(1-x)^{k-N}-(N-k)\left(\begin{array}{c}
N-1 \\
k-1
\end{array}\right) \int_{0}^{1}(1-x s)^{-N}(1-s)^{k-1} d s x^{k},
\end{aligned}
$$

откуда следует (3.6).

Докажем теперь некоторые вспомогательные оценки.

Лемма 3.1. Для $x \in \alpha_{2}$

$$
\left|R_{1}(x)\right| \leqslant \frac{q(x)^{4 L} \tau(x)}{2 L(1-\tau(x))^{2}}
$$

где $\alpha_{2}, R_{1}$ определены в (2.1), (2.3),

$$
q(x):=\left|\frac{x}{3 / 4}\right|^{3 / 4}\left|\frac{1-x}{1 / 4}\right|^{1 / 4}, \quad \tau(x):=\frac{|x|}{3|1-x|} .
$$

ДОКАЗАТЕЛЬСТВо. Если $x \in \alpha_{2}$, то

$$
q(x)=\left|\frac{x}{3 / 4}\right|^{3 / 4}\left|\frac{1-x}{1 / 4}\right|^{1 / 4}=\frac{|1-x|}{1 / 4}\left(\frac{|x|}{3|1-x|}\right)^{3 / 4}<1,
$$

откуда

$$
\tau(x)=\frac{|x|}{3|1-x|}=\left(q(x) \frac{1 / 4}{|1-x|}\right)^{4 / 3}<1,
$$

так как $|1-x|>1 / 4$ для $x \in \alpha_{2}$.

Так как для любого $\lambda \in(0,1)$ вьполнена оценка $\left(\begin{array}{c}N \\ l\end{array}\right) \leqslant \lambda^{-l}(1-\lambda)^{l-N}$, то для $x \in \alpha_{2}$ и $l>3 L$ имеем

$$
\begin{aligned}
\left|\left(\begin{array}{c}
4 L \\
l
\end{array}\right) x^{l}(1-x)^{4 L-l}\right| & \leqslant\left|\frac{x}{3 / 4}\right|^{l}\left|\frac{1-x}{1 / 4}\right|^{4 L-l} \\
& =\left(\frac{|1-x|}{1 / 4}\right)^{4 L}\left(\frac{|x|}{3|1-x|}\right)^{3 L}\left(\frac{|x|}{3|1-x|}\right)^{l-3 L} \\
& =q(x)^{4 L} \tau(x)^{l-3 L}
\end{aligned}
$$


Поэтому

$$
\begin{aligned}
R_{L, 1}(x) & :=\sum_{l=3 L+1}^{4 L}\left(\frac{l}{2 L}-\frac{3}{2}\right) b_{4 L, l}(x) \leqslant q(x)^{4 L} \sum_{l=3 L+1}^{4 L}\left(\frac{l}{2 L}-\frac{3}{2}\right) \tau(x)^{l-3 L} \\
& =\frac{q(x)^{4 L}}{2 L} \sum_{l=1}^{L} l \tau(x)^{l}=\frac{q(x)^{4 L} \tau(x)\left(1-(L+1) \tau(x)^{L}+L \tau(x)^{L+1}\right)}{2 L(1-\tau(x))^{2}} \\
& <\frac{q(x)^{4 L} \tau(x)}{2 L(1-\tau(x))^{2}} .
\end{aligned}
$$

Доказательство леммы 3.1 основано на идеях статьи [12].

СлЕДСтВИЕ 3.3. Для $|x| \leqslant 1 / 4$ выполнена оценка

$$
\left|R_{1}(x)\right|<\frac{9}{128 L}\left(\frac{5}{27}\right)^{L} .
$$

ДокАЗАТЕЛЬСТВО. Если $|x| \leqslant 1 / 4$, то $3 / 4 \leqslant|1-x| \leqslant 5 / 4$. Поэтому $q(x) \leqslant(5 / 27)^{1 / 4}$, $\tau(x) \leqslant 1 / 9$.

Следуюшие ниже оценки $B_{L}(1 / 4), L \in \mathbb{N}$, основаны на представлении некоторых многочленов Бернштейна при помоши неполной бета-функиии:

$$
\operatorname{Inc}_{a, b}(x):=\frac{\Gamma(a+b)}{\Gamma(a) \Gamma(b)} \int_{0}^{x} s^{a-1}(1-s)^{b-1} d s .
$$

Теорема 3.2. Для любого $N \in \mathbb{N} u$ любого $k \in\{0,1,2, \ldots, N\}$

$$
A_{N, k}(x)=1-\operatorname{Inc}_{k+1, N-k}(x) .
$$

ДокАЗАТЕЛЬСтво. Известно, что $\left(b_{N, k}(x)\right)^{\prime}=N\left(b_{N-1, k-1}(x)-b_{N-1, k}(x)\right)$, где $b_{N, l}(x)$ полагается равньм нулю при $l<0$ или $l>N$. Поэтому $\left(A_{N, k}(x)\right)^{\prime}=$ $-N b_{N-1, k}(x)$. Так как $A_{N, k}(0)=1$, то

$$
A_{N, k}(x)=1-N \int_{0}^{x} b_{N-1, k}(s) d s,
$$

откуда следует (3.9).

СЛЕДСТВИЕ 3.4. Имеет место равенство

$$
B_{L, 2}(x)=\frac{1}{2}-2 x+2 x \operatorname{Inc}_{L, 3 L}(x)-\frac{1}{2} \operatorname{Inc}_{L+1,3 L}(x) .
$$

ДоКАЗАТЕЛЬСТво. Легко видеть, что

$$
B_{L, 2}(x)=\frac{1}{2} A_{4 L, L}(x)-2 C_{4 L, L}(x)=\frac{1}{2} A_{4 L, L}(x)-2 x A_{4 L-1, L-1}(x),
$$

откуда при помощи (3.9) получаем (3.10). 
Лемма 3.2. Имеет место соотношение

$$
B_{L, 2}(1 / 4)=\frac{\sqrt{3}}{4 \sqrt{2 \pi L}} \Phi(L)<\frac{\sqrt{3}}{4 \sqrt{2 \pi L}}
$$

əде

$$
\Phi(L):=\frac{\Gamma^{*}(4 L)}{\Gamma^{*}(L) \Gamma^{*}(3 L)}, \quad \Gamma^{*}(r):=\sqrt{\frac{r}{2 \pi}} e^{r} r^{-r} \Gamma(r) .
$$

ДоКАЗАТЕЛЬСТво. На основании (3.10)

$$
\begin{aligned}
B_{L, 2}\left(\frac{1}{4}\right) & =\frac{1}{2}\left(\operatorname{Inc}_{L, 3 L}\left(\frac{1}{4}\right)-\operatorname{Inc}_{L+1,3 L}\left(\frac{1}{4}\right)\right) \\
& =\frac{(4 L-1) !}{(2 L-1) !(3 L-1) !} \int_{0}^{1 / 4}(1-4 s) s^{L-1}(1-s)^{3 L-1} d s
\end{aligned}
$$

Сделаем замену переменных (см. [13])

$$
-\frac{\zeta^{2}}{2}=\ln \left[\left(\frac{s}{1 / 4}\right)^{1 / 4}\left(\frac{1-s}{3 / 4}\right)^{3 / 4}\right]
$$

где знак $\zeta$ отрицательньй. Тогда

$$
B_{L, 2}\left(\frac{1}{4}\right)=\sqrt{\frac{3 L}{2 \pi}} \Phi(L) \int_{0}^{\infty} \exp \left(-2 L \zeta^{2}\right) \zeta d \zeta=\frac{\sqrt{3}}{4 \sqrt{2 \pi L}} \Phi(L) .
$$

Известно, что $\Gamma^{*}(r) \searrow 1$ при $r \rightarrow \infty$ и $\Phi(L) \nearrow 1$ при $L \rightarrow \infty$.

Лемма 3.3. Пусть $k, N \in \mathbb{N}, \delta>0, k<N-2$, Re $x<(k+1) /(N-1)$, $|x-(k+1) /(N-1)|>\delta$. Тогда

$$
\operatorname{In}_{N, k}(x):=\int_{0}^{1}(1-x s)^{-N}(1-s)^{k} d s=\frac{1}{k+1-(N-1) x}+O\left(k^{-3}\right) .
$$

ДокАЗАТЕЛЬСтво. Пусть $a:=(N-1) /(k+1)$. В интеграле $\operatorname{In}_{N, k}$ сделаем замену переменных $w:=w(s):=(1-s) /(1-x s)^{a}$. Тогда

$$
\frac{d w}{d s}=\frac{(a-(a-1) s) x-1}{(1-x s)^{a+1}}
$$

и

$$
\operatorname{In}_{N, k}(x)=\int_{0}^{1} \frac{w^{k}}{1-(a-(a-1) s) x} d w .
$$

Докажем, что $|w(s)|<1$ при достаточно малых $s \in[0,1]$. Пусть $x:=\rho e^{i \varphi}$. Тогда

$$
|w(s)|=\frac{1-s}{\left(1-2 \rho s \cos \varphi+\rho^{2} s^{2}\right)^{a / 2}} .
$$


Поэтому неравенство $|w(s)|<1$ эквивалентно

$$
\frac{\left(1-2 \rho s \cos \varphi+\rho^{2} s^{2}\right)^{a / 2}-1}{s}=\frac{f(s)-f(0)}{s}>-1
$$

где $f(s):=\left(1-2 \rho s \cos \varphi+\rho^{2} s^{2}\right)^{a / 2}$. Последнее неравенство вьполнено для достаточно малых $s \in[0,1]$, так как $f^{\prime}(0)=-a \rho \cos \varphi>-1$.

Пусть константа $\varepsilon \in(0,1)$ выбрана так, что $\varepsilon<\delta^{2} /\left((k+1)^{2}(a-1)|x|\right)$ и $|\tau|<1$, где $\tau:=w(\varepsilon)$. Тогда

$$
\operatorname{In}_{N, k}(x)=\int_{0}^{\tau} \frac{w^{k}}{1-(a-(a-1) s) x} d w+\int_{\tau}^{1} \frac{w^{k}}{1-(a-(a-1) s) x} d w=: I_{1}+I_{2}
$$

Так как $s \in[0,1]$, то $(a-(a-1) s) \in[1, a]$. Ясно, что отрезок $[x, a x]$ не пересекает круг $\{z:|1-z| \leqslant \delta\}$, поэтому $|1-(a-(a-1) s) x|>\delta$ для любого $s \in[0,1]$ и

$$
\left|I_{1}\right|<\frac{|\tau|^{k+1}}{(k+1) \delta}
$$

Для $I_{2}$ имеем

$$
\begin{aligned}
I_{2} & =\frac{1}{1-a x} \int_{\tau}^{1} w^{k} d w-\int_{\tau}^{1} \frac{w^{k}(a-1) x s}{(1-a x)(1-(a-(a-1) s) x)} d w \\
& =\frac{1-\tau^{k+1}}{(1-a x)(k+1)}-\int_{\tau}^{1} \frac{w^{k}(a-1) x s}{(1-a x)(1-(a-(a-1) s) x)} d w .
\end{aligned}
$$

Окончательно получаем

$$
\left|\operatorname{In}_{N, k}(x)-\frac{1}{(1-a x)(k+1)}\right|<\frac{2|\tau|^{k+1}}{\delta(k+1)}+\frac{(a-1)|x| \varepsilon}{(k+1) \delta^{2}}<\frac{2|\tau|^{k+1}}{\delta(k+1)}+\frac{1}{(k+1)^{3}}
$$

СЛЕДСТВИЕ 3.5. В условиях леммы 3.3 выполнено равенство

$$
\begin{aligned}
I_{N, k}(x) & =\operatorname{In}_{N, k}(x)-\operatorname{In}_{N, k+1}(x)=\frac{1}{(k+1-(N-1) x)(k+2-(N-1) x)}+O\left(k^{-3}\right) \\
& =\frac{1}{(k+1)^{2}\left(1-\frac{N-1}{k+1} x\right)^{2}}+O\left(k^{-3}\right) .
\end{aligned}
$$




\section{СПИСОК ЦИТИРОВАННОЙ ЛИТЕРАТУРЫ}

[1] Daubechies I. Orthonormal basis of compactly supported wavelets // Comm. Pure Appl. Math. 1988. V. 46. P. 909-996.

[2] Chui C.K., Wang J. High-Order Orthonormal Scaling Functions and Wavelets Give Poor Time-Frequency Localization. CAT Report \# 322, 1994.

[3] Novikov I. Ya. Modified Daubechies wavelets preserving localization with growth of smoothness // East J. Appr. 1995. V. 1. № 3. P. 341-348.

[4] Новиков И. Я. Константы неопределенности для модифицированных всплесков Добеши // Изв. Тул. гос. ун-та. Сер. Матем. Мех. Информатика. Т. 4. Вып. 1 Тула: ТулГУ, 1998. C. $107-111$.

[5] Бернштейн C. Demonstration de theoréme de Weierstrass fondée sur le calcul des probabilities // Сообщ. Харьковского матем. общества. Сер. 2. 1912-1913. Т. 13. №1. С. 1-2.

[6] Полиа Г., Сегё Г. Задачи и теоремы из анализа. Т. 2. М.: Наука, 1978.

[7] Shen J., Strang G. Asymptotics of Daubechies filters, scaling functions, and wavelets // Applied and Comp. Harm. Analysis. 1998. V. 5. P. 312-331.

[8] Kateb D., Lemarie-Rieusset P. G. The phase of the Daubechies filters // Université de Paris-Sud Mathématiques. Preprint 62, 1994. P. 1-42.

[9] Shen J., Strang G. The Zeros of the Daubechies Polynomials // Massachusetts Institute of Technology. Preprint, 1995. P. 1-12.

[10] Temme N.M. Asymptotics and Numerics of Zeros of Polynomials that are Related to Daubechies Wavelets // Centrum voor Wiskunde en Informatica. Preprint AM-R9613, 1996. P. 1-12.

[11] Jentzsch R. Untersuchungen zur Theorie der Folgen analytischer Functionen. Berlin: Inaug.-diss, 1914.

[12] Канторович Л. В. О сходимости последовательности полиномов Бернштейназа пределами основного интервала // Изв. АН СССР. 1931. С. 1103-1115.

[13] Temme N. M. Asymptotic inversion of the incomplete beta-function // J. Comp. Appl. Math. 1992. V. 41. P. 145-157. 